\title{
A FULL BAYESIAN APPROACH FOR INVERSE PROBLEMS
}

\author{
ALI MOHAMMAD-DJAFARI \\ Laboratoire des Signaux et Systèmes (CNRS-ESE-UPS), \\ Supélec, Plateau de Moulon, 91192 Gif-sur-Yvette, France ${ }^{\dagger}$
}

\begin{abstract}
The main object of this paper is to present some general concepts of Bayesian inference and more specifically the estimation of the hyperparameters in inverse problems. We consider a general linear situation where we are given some data $\boldsymbol{y}$ related to the unknown parameters $\boldsymbol{x}$ by $\boldsymbol{y}=\boldsymbol{A} \boldsymbol{x}+\boldsymbol{n}$ and where we can assign the probability laws $p(\boldsymbol{x} \mid \boldsymbol{\theta}), p(\boldsymbol{y} \mid \boldsymbol{x}, \boldsymbol{\beta}), p(\boldsymbol{\beta})$ and $p(\boldsymbol{\theta})$. The main discussion is then how to infer $\boldsymbol{x}, \boldsymbol{\theta}$ and $\boldsymbol{\beta}$ either individually or any combinations of them. Different situations are considered and discussed. As an important example, we consider the case where $\theta$ and $\beta$ are the precision parameters of the Gaussian laws to whom we assign Gamma priors and we propose some new and practical algorithms to estimate them simultaneously. Comparisons and links with other classical methods such as maximum likelihood are presented.
\end{abstract}

Key words: Bayesian inference, Hyperparameter estimation, Inverse problems, Maximum likelihood

\section{Introduction}

In a general Bayesian inference, we have the data $\boldsymbol{y}$, a known relation between the unknown parameters $\boldsymbol{x}$ and $\boldsymbol{y}$ and finally the hyperparameters $\boldsymbol{\beta}$ and $\boldsymbol{\theta}$. The Bayesian estimation technique is now well established [1-7] and has been used since many years to resolve the inverse problems in signal and image reconstruction and restoration [10-14,17,18,20,21].

The first step before applying the Bayes' rule is to assign the prior probability laws $p(\boldsymbol{x} \mid \boldsymbol{\theta}), p(\boldsymbol{y} \mid \boldsymbol{x}, \boldsymbol{\beta}), p(\boldsymbol{\theta})$ and $p(\boldsymbol{\beta})$. The next step is to determine the posterior laws and then to infer the unknowns. In this paper we are focusing more on the second step than on the first step. So we assume that all the direct probability laws are known.

The main object of this paper is to show how can we infer simultaneously the unknown parameters $\boldsymbol{x}$ and the hyperparameters $\boldsymbol{\beta}$ and $\boldsymbol{\theta}$ from the data $\boldsymbol{y}$.

\footnotetext{
${ }^{\dagger}$ Email: djafari@lss.supelec.fr
} 
Before going more in details let us give one example. This will permit us to fix the situations. Consider the case where the unknown parameters $\boldsymbol{x}$ represent the pixel values of an unobserved image and the data $\boldsymbol{y}$ are the pixel values of an observed image which is assumed to be a degraded version of it. If we consider a linear degradation we have

$$
\boldsymbol{y}=\boldsymbol{A x}+\boldsymbol{n},
$$

where $\boldsymbol{A}$ is a $(m \times n)$ matrix representing the degradation process and $\boldsymbol{n}$ represents the measurement uncertainty (noise) which is assumed to be additive, centered, white, Gaussian and independent of $\boldsymbol{x}$. This hypothesis leads us to

$$
p(\boldsymbol{y} \mid \boldsymbol{x}, \beta)=\frac{1}{Z_{1}(\beta)} \exp \left\{-\frac{1}{2} \beta(\boldsymbol{y}-\boldsymbol{A} \boldsymbol{x})^{t}(\boldsymbol{y}-\boldsymbol{A} \boldsymbol{x})\right\} .
$$

In this case $\beta$ is a positive parameter which is related to the noise variance $\sigma_{b}^{2}$ by $\beta=1 / \sigma_{b}^{2}$ and $Z_{1}(\beta)=(2 \beta / \pi)^{m / 2}$ is the normalizing factor.

Consider also, for this example, a Gaussian prior law for $\boldsymbol{x}$ :

$$
p(\boldsymbol{x} \mid \theta)=\frac{1}{Z_{2}(\theta)} \exp \left\{-\frac{1}{2} \theta \phi(\boldsymbol{x})\right\} \quad \text { with } \quad \phi(\boldsymbol{x})=\boldsymbol{x}^{t} \boldsymbol{P}_{0}^{-1} \boldsymbol{x},
$$

where $\theta=1 / \sigma_{x}^{2}$ is a positive parameter, $\boldsymbol{P}_{0}$ is the a priori covariance matrix of $\boldsymbol{x}$ and $Z_{2}(\theta)=(2 \theta / \pi)^{n / 2}\left|\boldsymbol{P}_{0}\right|^{1 / 2}$.

A well known case is the situation where $\theta, \beta$ and $\boldsymbol{P}_{0}$ are known and we only want to estimate $\boldsymbol{x}$. In fact, in this special case, the joint law $p(\boldsymbol{y}, \boldsymbol{x} \mid \boldsymbol{\theta}, \boldsymbol{\beta})$ and the posterior law $p(\boldsymbol{x} \mid \boldsymbol{y}, \boldsymbol{\theta}, \boldsymbol{\beta})$ are both Gaussian and we have

$$
p(\boldsymbol{x} \mid \boldsymbol{y}, \boldsymbol{\theta}, \boldsymbol{\beta}) \propto \exp \left\{-\frac{1}{2} \beta(\boldsymbol{y}-\boldsymbol{A} \boldsymbol{x})^{t}(\boldsymbol{y}-\boldsymbol{A} \boldsymbol{x})-\frac{1}{2} \theta \boldsymbol{x}^{t} \boldsymbol{P}_{0}^{-1} \boldsymbol{x}\right\},
$$

and, if we note by

$$
\widehat{\boldsymbol{x}}=\arg \min _{\boldsymbol{x}}\left\{J(\boldsymbol{x})=(\boldsymbol{y}-\boldsymbol{A x})^{t}(\boldsymbol{y}-\boldsymbol{A x})-\lambda \boldsymbol{x}^{t} \boldsymbol{P}_{0}^{-1} \boldsymbol{x}\right\} \text { with } \lambda=\theta / \beta,
$$

then, it is easy to show that

$$
\boldsymbol{x} \mid \boldsymbol{y} \sim \mathcal{N}(\widehat{\boldsymbol{x}}, \widehat{\boldsymbol{P}}) \text { with }\left\{\begin{array}{l}
\widehat{\boldsymbol{x}}=\beta \widehat{\boldsymbol{P}} \boldsymbol{A}^{t} \boldsymbol{y} \\
\widehat{\boldsymbol{P}}=\beta^{-1}\left(\boldsymbol{A}^{t} \boldsymbol{A}+\lambda \boldsymbol{P}_{0}^{-1}\right)^{-1}
\end{array}\right.
$$

One can make a comparison with the classical regularization techniques for inverse problems with smoothness hypothesis, where $\boldsymbol{P}_{0}^{-1}=\boldsymbol{D}^{t} \boldsymbol{D}$ with $\boldsymbol{D}$ a matrix approximating a differentiation operator and $\lambda$ is called the regularization parameter [14].

What we address here is the generalization of the problem of the determination of the regularization parameter $\lambda$ which has been studied for a long time [22$28,15,18,30,31]$ and is still an open problem.

What is proposed here is to consider the general case where $\boldsymbol{\theta}$ and $\boldsymbol{\beta}$ are considered to be unknown and we are facing to make inference as well about $\boldsymbol{x}$ 
as about them. What we propose is to consider the hyperparameters $\boldsymbol{\theta}$ and $\boldsymbol{\beta}$ in the same manner than $\boldsymbol{x}$, i.e; translate our prior knowledge about them by the probability laws $p(\boldsymbol{\theta})$ and $p(\boldsymbol{\beta})$, then determine the posterior laws and finally infer about them from these posterior laws.

\section{General Bayesian inference approach}

Assume now that we know the expressions of all the prior laws. We can then calculate the joint probability law:

$$
p(\boldsymbol{y}, \boldsymbol{x}, \boldsymbol{\theta}, \boldsymbol{\beta})=p(\boldsymbol{y} \mid \boldsymbol{x}, \boldsymbol{\beta}) p(\boldsymbol{x} \mid \boldsymbol{\theta}) p(\boldsymbol{\theta}) p(\boldsymbol{\beta}) .
$$

In an ideal case where we are given $\boldsymbol{A}, \boldsymbol{y}, \boldsymbol{\beta}$ and $\boldsymbol{\theta}$, to infer $\boldsymbol{x}$ we can calculate the posterior law $p(\boldsymbol{x} \mid \boldsymbol{y}, \boldsymbol{\theta}, \boldsymbol{\beta})$ and if we choose as the solution to our problem the Maximum a posteriori (MAP) estimate, we have:

$$
\widehat{\boldsymbol{x}}=\arg \max _{\boldsymbol{x}}\{p(\boldsymbol{x} \mid \boldsymbol{y}, \boldsymbol{\theta}, \boldsymbol{\beta})\}=\arg \max _{\boldsymbol{x}}\{p(\boldsymbol{y} \mid \boldsymbol{x}, \boldsymbol{\beta}) p(\boldsymbol{x} \mid \boldsymbol{\theta})\} .
$$

But, unfortunately, in practical situations we are not given $\boldsymbol{\beta}$ and $\boldsymbol{\theta}$ and the main problem is how to infer them. We consider the following situations:

1. The first is to estimate the three quantities simultaneously. We call this method Joint Maximum a posteriori (JMAP) and the estimates are defined as

$$
(\widehat{\boldsymbol{x}}, \widehat{\boldsymbol{\theta}}, \widehat{\boldsymbol{\beta}})=\arg \max _{(\boldsymbol{x}, \boldsymbol{\theta}, \boldsymbol{\beta})}\{p(\boldsymbol{y} \mid \boldsymbol{x}, \boldsymbol{\beta}) p(\boldsymbol{x} \mid \boldsymbol{\theta}) p(\boldsymbol{\beta}) p(\boldsymbol{\theta})\} .
$$

One practical way to do this joint optimization is to use the following algorithm

$$
\left\{\begin{array}{l}
\widehat{\boldsymbol{x}}^{k+1}=\arg \max _{\boldsymbol{x}}\left\{p\left(\boldsymbol{y} \mid \boldsymbol{x}, \widehat{\boldsymbol{\beta}}^{k}\right) p\left(\boldsymbol{x} \mid \widehat{\boldsymbol{\theta}}^{k}\right)\right\} \\
\widehat{\boldsymbol{\theta}}^{k+1}=\arg \max _{\boldsymbol{\theta}}\left\{p\left(\widehat{\boldsymbol{x}}^{k} \mid \boldsymbol{\theta}\right) p(\boldsymbol{\theta})\right\} \\
\widehat{\boldsymbol{\beta}}^{k+1}=\arg \max _{\boldsymbol{\beta}}\left\{p\left(\boldsymbol{y} \mid \widehat{\boldsymbol{x}}^{k}, \boldsymbol{\beta}\right) p(\boldsymbol{\beta})\right\}
\end{array}\right.
$$

2. In the second case $\boldsymbol{\theta}$ and $\boldsymbol{\beta}$ are considered as the nuisance parameters and are integrated out of the problem and $\boldsymbol{x}$ is estimated by

$$
\begin{aligned}
\widehat{\boldsymbol{x}} & =\arg \max _{\boldsymbol{x}}\{p(\boldsymbol{x} \mid \boldsymbol{y})\}=\arg \max _{\boldsymbol{x}}\left\{\int p(\boldsymbol{y}, \boldsymbol{x}, \boldsymbol{\theta}, \boldsymbol{\beta}) \mathrm{d} \boldsymbol{\theta} \mathrm{d} \boldsymbol{\beta}\right\} \\
& =\arg \max _{\boldsymbol{x}}\left\{\int p(\boldsymbol{y} \mid \boldsymbol{x}, \boldsymbol{\beta}) p(\boldsymbol{\beta}) \mathrm{d} \boldsymbol{\beta} \int p(\boldsymbol{x} \mid \boldsymbol{\theta}) p(\boldsymbol{\theta}) \mathrm{d} \boldsymbol{\theta}\right\} .
\end{aligned}
$$

We call this method Marginalized MAP type one $\left(\mathrm{MMAP}^{1}\right)$.

3 . In the third case only $\boldsymbol{\theta}$ is considered as the nuisance parameter and is integrated out of the problem and $\boldsymbol{x}$ and $\boldsymbol{\beta}$ are estimated by

$$
\begin{aligned}
(\widehat{\boldsymbol{x}}, \widehat{\boldsymbol{\beta}}) & =\arg \max _{(\boldsymbol{x}, \boldsymbol{\beta})}\{p(\boldsymbol{x}, \boldsymbol{\beta} \mid \boldsymbol{y}, \boldsymbol{\theta})\}=\arg \max _{(\boldsymbol{x}, \boldsymbol{\beta})}\left\{\int p(\boldsymbol{y}, \boldsymbol{x}, \boldsymbol{\theta}, \boldsymbol{\beta}) \mathrm{d} \boldsymbol{\theta}\right\} \\
& =\arg \max _{(\boldsymbol{x}, \boldsymbol{\beta})}\left\{p(\boldsymbol{y} \mid \boldsymbol{x}, \boldsymbol{\beta}) p(\boldsymbol{\beta}) \int p(\boldsymbol{x} \mid \boldsymbol{\theta}) p(\boldsymbol{\theta}) \mathrm{d} \boldsymbol{\theta}\right\} .
\end{aligned}
$$


We call this method Marginalized MAP type two $\left(\mathrm{MMAP}^{2}\right)$.

4. Finally, in the last case we may first estimate $\widehat{\boldsymbol{\theta}}$ and $\widehat{\boldsymbol{\beta}}$ by

$$
\begin{aligned}
(\widehat{\boldsymbol{\theta}}, \widehat{\boldsymbol{\beta}}) & =\arg \max _{(\boldsymbol{\theta}, \widehat{\boldsymbol{\beta}})}\{p(\boldsymbol{\theta}, \boldsymbol{\beta} \mid \boldsymbol{y})\}=\arg \max _{\boldsymbol{\theta}, \boldsymbol{\beta})}\left\{\int p(\boldsymbol{y}, \boldsymbol{x}, \boldsymbol{\theta}, \boldsymbol{\beta}) \mathrm{d} \boldsymbol{x}\right\} \\
& =\arg \max _{(\boldsymbol{\theta}, \widehat{\boldsymbol{\beta}})}\left\{p(\boldsymbol{\beta}) p(\boldsymbol{\theta}) \int p(\boldsymbol{y} \mid \boldsymbol{x}, \boldsymbol{\beta}) p(\boldsymbol{x} \mid \boldsymbol{\theta}) \mathrm{d} \boldsymbol{x}\right\} \\
& =\arg \max _{(\boldsymbol{\theta}, \boldsymbol{\beta})}\{p(\boldsymbol{\beta}) p(\boldsymbol{\theta}) l(\boldsymbol{\theta}, \boldsymbol{\beta} \mid \boldsymbol{y})\} .
\end{aligned}
$$

and then used them for the estimation of $\boldsymbol{x}$ by

$$
\widehat{\boldsymbol{x}}=\arg \max _{\boldsymbol{x}}\{p(\boldsymbol{x} \mid \boldsymbol{y}, \widehat{\boldsymbol{\theta}}, \widehat{\boldsymbol{\beta}})\} .
$$

We call this method Marginalized MAP type three $\left(\mathrm{MMAP}^{3}\right)$.

Note that if $p(\boldsymbol{\theta})$ and $p(\boldsymbol{\beta})$ are uniform functions of $\boldsymbol{\theta}$ and $\boldsymbol{\beta}$, then $\widehat{\boldsymbol{\theta}}$ and $\widehat{\boldsymbol{\beta}}$ correspond to the classical maximum likelihood (ML) estimates because $l(\boldsymbol{\theta}, \boldsymbol{\beta} \mid \boldsymbol{y})$ is, for a given $\boldsymbol{y}$, the likelihood function of $\boldsymbol{\theta}$ and $\boldsymbol{\beta}$.

The calculus of $l(\boldsymbol{\theta}, \boldsymbol{\beta} \mid \boldsymbol{y})$ is not easy and so is its optimization. Many works have been done on the subject. We distinguish three kind of methods:

- The first is to use the Expectation-Maximization (EM) algorithm which has been developed exactly in the context of ML parameter estimation $[32,4,33]$.

- The second is to estimate the integral using a Monte Carlo simulation method (Stochastic EM: SEM).

- The third is to make some approximations. For example, at each iteration during the optimization, one may obtain an analytical expression for that integral by approximating the expression inside it by a second order polynomial (Gaussian quadrature approximation).

We will consider this last method.

\section{A case study}

Let us consider the following simple linear inverse problem $\boldsymbol{y}=\boldsymbol{A x}+\boldsymbol{n}$ and make the following hypothesis:

- The noise $\boldsymbol{n}$ is considered to be white, centered and Gaussian with precision $\beta$, so that we have

$$
\boldsymbol{y} \mid \boldsymbol{x}, \beta \sim \mathcal{N}\left(\boldsymbol{A} \boldsymbol{x}, \beta^{-1} \boldsymbol{I}\right) \longrightarrow p(\boldsymbol{y} \mid \boldsymbol{x}, \beta)=\frac{1}{Z_{1}(\beta)} \exp \left\{-\frac{1}{2} \beta\|\boldsymbol{y}-\boldsymbol{A} \boldsymbol{x}\|^{2}\right\} .
$$

where $Z_{1}(\beta) \propto \beta^{m / 2}$.

- Our prior prior knowledge about $\boldsymbol{x}$ can be translated by

$$
p(\boldsymbol{x} \mid \theta)=\frac{1}{Z_{2}(\theta)} \exp \left\{-\frac{1}{2} \theta \phi(\boldsymbol{x})\right\} .
$$

where we will consider the following special cases for $\phi(\boldsymbol{x})$ : 
- Gaussian priors:

$$
\phi_{G}(\boldsymbol{x})=\boldsymbol{x}^{t} \boldsymbol{P}_{0}^{-1} \boldsymbol{x}=\|\boldsymbol{D} \boldsymbol{x}\|^{2} \longrightarrow \boldsymbol{x} \mid \theta \sim \mathcal{N}\left(\mathbf{0}, \theta^{-1} \boldsymbol{P}_{0}^{-1}\right),
$$

which can also be written $\phi_{G}(\boldsymbol{x})=\sum_{j} \sum_{i} p_{i j} x_{i} x_{j}$ with some special cases:

$$
\phi_{G}(\boldsymbol{x})=\sum_{j} x_{j}^{2}, \quad \text { or } \quad \phi_{G}(\boldsymbol{x})=\sum_{j}\left|x_{j}-x_{j-1}\right|^{2} .
$$

- Generalized Gaussian priors:

$$
\phi_{G G}(\boldsymbol{x})=\sum_{j}\left|x_{j}-x_{j-1}\right|^{p}, \quad 1<p \leq 2
$$

- Entropic priors:

$$
\phi_{E}(\boldsymbol{x})=\sum_{j=1}^{n} S\left(x_{j}\right) \text { where } S\left(x_{j}\right)=\left\{x_{j}^{2}, x_{j} \ln x_{j}-x_{j}, \ln x_{j}-x_{j}\right\} .
$$

- Markovian priors:

$$
\phi_{M}(\boldsymbol{x})=\sum_{j} \sum_{i \in N_{j}} V\left(x_{j}, x_{i}\right), \quad \text { where } V\left(x_{j}, x_{i}\right) \text { is a potential function }
$$

and where $N_{j}$ is a set of sites considered to be neighbors of site $j$, for example $N_{j}=\{j-1, j+1\}, \quad$ or $\quad N_{j}=\{j-2, j-1, j+1, j+2\}$.

Note that, in all cases $\theta$ is generally a positive parameter. Note also that in the first case we have $Z_{2}(\theta) \propto \theta^{n / 2}$. Unfortunately we have not an analytic expression for $Z_{2}(\theta)$ in the other cases. However, in the situations we are concerned with, $Z_{2}(\theta)$ can either be calculated numerically or approximated by

$$
Z_{2}(\theta) \propto \theta^{\alpha n / 2}
$$

$-\theta$ and $\beta$ are both positive parameters. We choose Gamma prior laws for them:

$$
\begin{array}{ll}
\theta \sim \mathcal{G}(a, \zeta) \longrightarrow p(\theta) \propto \theta^{(a-1)} \exp \{-\zeta \theta\} \longrightarrow \mathrm{E}\{\theta\}=a / \zeta, & \operatorname{Var}\{\theta\}=a / \zeta^{2} \\
\beta \sim \mathcal{G}(b, \zeta) \longrightarrow p(\beta) \propto \beta^{(b-1)} \exp \{-\zeta \beta\} \longrightarrow \mathrm{E}\{\beta\}=b / \zeta, & \operatorname{Var}\{\beta\}=b / \zeta^{2}
\end{array}
$$

Now, using the following notations

$$
\begin{gathered}
Q(\boldsymbol{x})=\|\boldsymbol{y}-\boldsymbol{A} \boldsymbol{x}\|^{2}, \quad J_{0}(\boldsymbol{x})=\beta Q(\boldsymbol{x})+\theta \phi(\boldsymbol{x}), \\
\nabla Q(\boldsymbol{x})=-2 \boldsymbol{A}^{t}(\boldsymbol{y}-\boldsymbol{A} \boldsymbol{x}), \quad \text { and } \quad \nabla J_{0}(\boldsymbol{x})=\beta \nabla Q(\boldsymbol{x})+\theta \nabla \phi(\boldsymbol{x}),
\end{gathered}
$$

we can calculate the expression of the joint $p d f p(\boldsymbol{y}, \boldsymbol{x}, \theta, \beta)=p(\boldsymbol{y} \mid \boldsymbol{x}, \beta) p(\boldsymbol{x} \mid \theta) p(\theta) p(\beta)$, which can be written

$$
p(\boldsymbol{y}, \boldsymbol{x}, \theta, \beta) \propto \theta^{-(\alpha n / 2-a+1)} \beta^{-(m / 2-b+1)} \exp \left\{-\frac{1}{2} J_{1}(\boldsymbol{x})\right\},
$$


with

$$
J_{1}(\boldsymbol{x})=\beta[Q(\boldsymbol{x})+2 \zeta]+\theta[\phi(\boldsymbol{x})+2 \zeta]=J_{0}(\boldsymbol{x})+2 \zeta(\theta+\beta) .
$$

This will let us to go further in details of some of the above mentioned cases. For example in the Gaussian case we have:

$\boldsymbol{x} \mid \boldsymbol{y}, \theta, \beta \sim \mathcal{N}(\widehat{\boldsymbol{x}}, \widehat{\boldsymbol{P}})$ with $\widehat{\boldsymbol{x}}=\beta\left(\beta \boldsymbol{A}^{t} \boldsymbol{A}+\theta \boldsymbol{P}_{0}^{-1}\right)^{-1} \boldsymbol{A}^{t} \boldsymbol{y}$ and $\widehat{\boldsymbol{P}}=\left(\beta \boldsymbol{A}^{t} \boldsymbol{A}+\theta \boldsymbol{P}_{0}^{-1}\right)^{-1}$

$$
\begin{aligned}
\theta \mid \boldsymbol{y}, \boldsymbol{x}, \beta & \sim \mathcal{G}\left(a-\alpha n / 2, \frac{1}{2}[\phi(\boldsymbol{x})+2 \zeta]\right) \longrightarrow \mathrm{E}\{\theta \mid \boldsymbol{y}, \boldsymbol{x}, \beta\}=\frac{2 a-\alpha n}{[\phi(\boldsymbol{x})+2 \zeta]} \\
\beta \mid \boldsymbol{y}, \boldsymbol{x}, \theta & \sim \mathcal{G}\left(b-m / 2, \frac{1}{2}[Q(\boldsymbol{x})+2 \zeta]\right) \longrightarrow \mathrm{E}\{\beta \mid \boldsymbol{y}, \boldsymbol{x}, \theta\}=\frac{2 b-m}{[Q(\boldsymbol{x})+2 \zeta]}
\end{aligned}
$$

Now, let us consider the four aforementioned methods a little more in details.

\subsection{JOINT MAXIMUM A POSTERIORI (JMAP)}

Using the expressions and the notations of the last paragraph in (11) we have to deal with the following algorithm:

$$
\begin{aligned}
\widehat{\boldsymbol{x}}^{k+1} & =\arg \max _{\boldsymbol{x}}\left\{p\left(\boldsymbol{y} \mid \boldsymbol{x}, \widehat{\beta}^{k}\right) p\left(\boldsymbol{x} \mid \widehat{\theta}^{k}\right)\right\}=\arg \min _{\boldsymbol{x}}\left\{J_{0}\left(\boldsymbol{x}, \widehat{\beta}^{k}, \widehat{\theta}^{k}\right)\right\}, \\
\widehat{\theta}^{k+1} & =\arg \max _{\theta}\left\{p\left(\widehat{\boldsymbol{x}}^{k} \mid \theta\right) p(\theta)\right\}=\arg \min _{\theta}\left\{\left[\phi\left(\widehat{\boldsymbol{x}}^{k}\right)+2 \zeta\right] \theta-(2 a-\alpha n-2) \ln \theta\right\}, \\
\widehat{\beta}^{k+1} & =\arg \max _{\beta}\left\{p\left(\boldsymbol{y} \mid \widehat{\boldsymbol{x}}^{k}, \beta\right) p(\beta)\right\}=\arg \min _{\beta}\left\{\left[Q\left(\widehat{\boldsymbol{x}}^{k}\right)+2 \zeta\right] \beta-(2 b-m-2) \ln \beta\right\} .
\end{aligned}
$$

The two last equations have explicit solutions. In the case of Gaussian priors, the first equation has also an explicit solution. However, in general, we propose the following gradient based algorithm:

$$
\text { Algorithm 1: } \begin{aligned}
\widehat{\boldsymbol{x}}^{k+1} & =(1-\mu) \widehat{\boldsymbol{x}}^{k}-\mu \nabla J_{0}\left(\widehat{\boldsymbol{x}}^{k}, \widehat{\beta}^{k}, \widehat{\theta}^{k}\right) \\
& =(1-\mu) \widehat{\boldsymbol{x}}^{k}-\mu\left[\widehat{\beta}^{k} \nabla Q\left(\widehat{\boldsymbol{x}}^{k}\right)+\widehat{\theta}^{k} \nabla \phi\left(\widehat{\boldsymbol{x}}^{k}\right)\right], \quad 0<\mu<1, \\
\widehat{\theta}^{k+1} & =\frac{(2 a-\alpha n-2)}{\left[\phi\left(\widehat{\boldsymbol{x}}^{k}\right)+2 \zeta\right]}, \quad a>(\alpha n+2) / 2, \\
\widehat{\beta}^{k+1} & =\frac{(2 b-m-2)}{\left[Q\left(\widehat{\boldsymbol{x}}^{k}\right)+2 \zeta\right]}, \quad b>(m+2) / 2 .
\end{aligned}
$$

The conditions $a>(\alpha n+2) / 2$ and $b>(m+2) / 2$ are added to satisfy, when necessary, the positivity constraint of $\widehat{\boldsymbol{\theta}}$ and $\widehat{\boldsymbol{\beta}}$.

\subsection{MARGINALIZED MAXIMUM A POSTERIORI MMAP ${ }^{1}$}

Considering $\theta$ and $\beta$ as the nuisance parameters and integrating out them from $p(\boldsymbol{y}, \boldsymbol{x}, \theta, \beta)$ we obtain

$$
p(\boldsymbol{y}, \boldsymbol{x})=\iint p(\boldsymbol{y}, \boldsymbol{x}, \theta, \beta) \mathrm{d} \beta \mathrm{d} \theta \propto[Q(\boldsymbol{x})+2 \zeta]^{-(m-2 b) / 2}[\phi(\boldsymbol{x})+2 \zeta]^{-(\alpha n-2 a) / 2}
$$


Now, defining $\quad \widehat{\boldsymbol{x}}_{\mathrm{MMAP}}=\arg \max _{\boldsymbol{x}}\{p(\boldsymbol{x} \mid \boldsymbol{y})\}=\arg \min _{\boldsymbol{x}}\left\{\frac{1}{2} J_{2}(\boldsymbol{x})\right\}$,

with $\quad J_{2}(\boldsymbol{x})=(2 a-\alpha n) \ln [Q(\boldsymbol{x})+2 \zeta]+(2 b-m) \ln [\phi(\boldsymbol{x})+2 \zeta]$,

and trying to calculate this solution by an iterative gradient based algorithm, we have to calculate

$$
\nabla J_{2}(\boldsymbol{x})=\frac{(2 a-\alpha n)}{[Q(\boldsymbol{x})+2 \zeta]} \nabla Q(\boldsymbol{x})+\frac{(2 b-m)}{[\phi(\boldsymbol{x})+2 \zeta]} \nabla \phi(\boldsymbol{x}) .
$$

We propose then the following iterative algorithm:

Algorithm 2: $\quad \widehat{\boldsymbol{x}}^{k+1}=(1-\mu) \widehat{\boldsymbol{x}}^{k}-\mu \nabla J_{2}\left(\widehat{\boldsymbol{x}}^{k}\right)$

$$
\begin{aligned}
& =(1-\mu) \widehat{\boldsymbol{x}}^{k}-\mu\left[\widehat{\beta}^{k} \nabla Q\left(\widehat{\boldsymbol{x}}^{k}\right)+\widehat{\theta}^{k} \nabla \phi\left(\widehat{\boldsymbol{x}}^{k}\right)\right], \quad 0<\mu<1, \\
\widehat{\theta}^{k} & =\frac{(2 a-\alpha n)}{\left[\phi\left(\widehat{\boldsymbol{x}}^{k}\right)+2 \zeta\right]}, \quad a>\alpha n / 2, \\
\widehat{\beta}^{k} & =\frac{(2 b-m)}{\left[Q\left(\widehat{\boldsymbol{x}}^{k}\right)+2 \zeta\right]}, \quad b>m / 2 .
\end{aligned}
$$

\subsection{MARGINALIZED MAXIMUM A POSTERIORI MMAP ${ }^{2}$}

In this case, $\theta$ only is considered as a nuisance parameter and is integrated out:

$$
\begin{aligned}
p(\boldsymbol{y}, \boldsymbol{x}, \beta) & =\int p(\boldsymbol{y}, \boldsymbol{x}, \theta, \beta) \mathrm{d} \theta \\
& \propto \beta^{-m / 2+b-1}[\phi(\boldsymbol{x})+2 \zeta]^{-(\alpha n-2 a) / 2} \exp \left\{-\frac{1}{2} \beta[Q(\boldsymbol{x})+2 \zeta]\right\} .
\end{aligned}
$$

Then, $\boldsymbol{x}$ and $\beta$ are estimated by

$$
(\widehat{\boldsymbol{x}}, \widehat{\beta})=\underset{\boldsymbol{x}, \beta}{\arg \max }\{p(\boldsymbol{y}, \boldsymbol{x}, \beta)\} .
$$

Noting

$$
-2 \ln p(\boldsymbol{y}, \boldsymbol{x}, \beta)=-(2 b-m-2) \ln \beta+(2 a-\alpha n) \ln [\phi(\boldsymbol{x})+2 \zeta]+\beta[Q(\boldsymbol{x})+2 \zeta]
$$

and differentiating it with respect to $\beta$ gives

$$
\widehat{\beta}=\frac{2 b-m-2}{[Q(\boldsymbol{x})+2 \zeta]} .
$$

So, noting

$$
\begin{aligned}
& J_{3}(\boldsymbol{x}, \beta)=(2 a-\alpha n) \ln [\phi(\boldsymbol{x})+2 \zeta]+\beta[Q(\boldsymbol{x})+2 \zeta] \\
& \text { and } \quad \nabla J_{3}(\boldsymbol{x}, \beta)=\frac{2 a-\alpha n}{[\phi(\boldsymbol{x})+2 \zeta]} \nabla \phi(\boldsymbol{x})+\beta \nabla Q(\boldsymbol{x}),
\end{aligned}
$$


and using a gradient based algorithm for minimizing $J_{3}$ with respect to $\boldsymbol{x}$ we propose the following:

$$
\begin{aligned}
\text { Algorithm 3: } \quad \widehat{\boldsymbol{x}}^{k+1} & =(1-\mu) \widehat{\boldsymbol{x}}^{k+1}-\widehat{\beta}^{k} \nabla Q(\boldsymbol{x})-\widehat{\theta}^{k} \nabla \phi(\boldsymbol{x}), \quad 0<\mu<1, \\
\widehat{\theta}^{k} & =\frac{2 a-\alpha n}{\left[\phi\left(\widehat{\boldsymbol{x}}^{k}\right)+2 \zeta\right]}, \quad a>(\alpha n+2) / 2 \\
\widehat{\beta}^{k} & =\frac{2 b-m-2}{\left[Q\left(\widehat{\boldsymbol{x}}^{k}\right)+2 \zeta\right]}, \quad b>(m+2) / 2 .
\end{aligned}
$$

\subsection{MAXIMUM LIKELIHOOD OR MMAP}

In this case first $\boldsymbol{x}$ integrated out $\boldsymbol{x}$ from $p(\boldsymbol{y}, \boldsymbol{x}, \theta, \beta)$ to obtain:

$$
\begin{aligned}
p(\boldsymbol{y}, \theta, \beta)= & \int p(\boldsymbol{y}, \boldsymbol{x}, \theta, \beta) \mathrm{d} \boldsymbol{x}=\frac{\beta^{(b-1)}}{Z_{2}(\beta)} \frac{\theta^{(a-1)}}{Z_{1}(\theta)} \int \exp \left\{-\frac{1}{2} J_{1}(\boldsymbol{x}, \beta, \theta)\right\} \mathrm{d} \boldsymbol{x} \\
& \text { with } \quad J_{1}(\boldsymbol{x}, \beta, \theta)=\beta[Q(\boldsymbol{x})+2 \zeta]+\theta[\phi(\boldsymbol{x})+2 \zeta] .
\end{aligned}
$$

Excepted the Gaussian case where $J_{1}$ is a quadratic function of $\boldsymbol{x}$, in general, it is not easy to obtain an analytical expression for this integral. One can then try to make a Gaussian approximation which means to develop $J_{1}$ around its minimum $\widehat{\boldsymbol{x}}_{\mathrm{MAP}}=\arg \min _{\boldsymbol{x}}\left\{J_{1}(\boldsymbol{x}, \beta, \theta)\right\}$ by

$$
J_{1}(\boldsymbol{x}, \beta, \theta) \simeq \frac{1}{2}\left(\boldsymbol{x}-\widehat{\boldsymbol{x}}_{\mathrm{MAP}}\right)^{t} \boldsymbol{M}\left(\boldsymbol{x}-\widehat{\boldsymbol{x}}_{\mathrm{MAP}}\right)+\boldsymbol{g}^{t}\left(\boldsymbol{x}-\widehat{\boldsymbol{x}}_{\mathrm{MAP}}\right)+c,
$$

where $\boldsymbol{g}=\beta \nabla Q(\boldsymbol{x})+\theta \nabla \phi(\boldsymbol{x})$ is the gradient of $J_{1}$ and $\boldsymbol{M}$ is its Hessian, both calculated for $\widehat{\boldsymbol{x}}_{\mathrm{MAP}}$. With this approximation we obtain

$$
p(\boldsymbol{y}, \theta, \beta)=\beta^{-m / 2+b-1} \theta^{-\alpha n / 2+a-1}|\boldsymbol{M}(\beta, \theta)|^{-\frac{1}{2}} \exp \left\{-\frac{1}{2} J_{1}\left(\widehat{\boldsymbol{x}}_{\mathrm{MAP}}, \beta, \theta\right)\right\} .
$$

Differentiating $l(\theta, \beta \mid \boldsymbol{y})=\ln p(\boldsymbol{y}, \theta, \beta)$ with respect to $\beta$ and $\theta$ gives

$$
\widehat{\beta}=\frac{2 b-m-2}{\left[Q\left(\widehat{\boldsymbol{x}}^{k}\right)+2 \zeta\right]+\operatorname{trace}\left[\boldsymbol{M}^{-1} \boldsymbol{A}^{t} \boldsymbol{A}\right]}, \quad \hat{\theta}=\frac{2 a-\alpha n-2}{\left[\phi\left(\widehat{\boldsymbol{x}}^{k}\right)+2 \zeta\right]+\operatorname{trace}\left[\boldsymbol{M}^{-1} \boldsymbol{P}_{0}^{-1}\right]} .
$$

where $\boldsymbol{P}_{0}^{-1}$ is the Hessian of $\phi(\boldsymbol{x}), \boldsymbol{A}^{t} \boldsymbol{A}$ is the Hessian of $Q(\boldsymbol{x})$ and $\boldsymbol{M}$ is the Hessian of $J_{1}(\boldsymbol{x})$ :

$$
\boldsymbol{M}(\beta, \theta)=\beta \boldsymbol{A}^{t} \boldsymbol{A}+\theta \boldsymbol{P}_{0}^{-1} .
$$

Using these expressions we propose the following algorithm:

$$
\begin{aligned}
\operatorname{Algorithm} 4: \widehat{\boldsymbol{x}}^{k} & =\underset{\boldsymbol{x}}{\arg \min }\left\{J_{1}\left(\boldsymbol{x}, \widehat{\beta}^{k}, \widehat{\theta}^{k}\right)\right\}=\boldsymbol{M}\left(\widehat{\beta}^{k}, \widehat{\theta}^{k}\right)^{-1} \boldsymbol{A}^{t} \boldsymbol{y} \\
\widehat{\theta}^{k+1} & =\frac{2 a-\alpha n-2}{\left[\phi\left(\widehat{\boldsymbol{x}}^{k}\right)+2 \zeta\right]+\operatorname{trace}\left[\boldsymbol{M}^{-1} \boldsymbol{P}_{0}^{-1}\right]} \\
\widehat{\beta}^{k+1} & =\frac{2 b-m-2}{\left[Q\left(\widehat{\boldsymbol{x}}^{k}\right)+2 \zeta\right]+\operatorname{trace}\left[\boldsymbol{M}^{-1} \boldsymbol{A}^{t} \boldsymbol{A}\right]}
\end{aligned}
$$


This algorithm needs the inversion of the matrix $\boldsymbol{M}$ which is very costly in practice.

\section{Comparison and the main structure of the proposed algorithmes}

Comparing the Algorithms 1 to $\mathbf{4}$, one can see that they all have the same structure:

- for fixed $\theta$ and $\beta$ optimize locally a criterion $J(\boldsymbol{x}, \beta, \theta)$, and

- update $\theta$ and $\beta$ using the solution $\widehat{\boldsymbol{x}}$ just obtained and iterate until convergence.

Note also that only in Algorithm 4, the updating step takes account of the measurement system operator $\boldsymbol{A}$ and the covariance structure $\boldsymbol{P}_{0}$ of the input $\boldsymbol{x}$.

\section{Conclusions and perspectives}

We considered the inverse problem of infering the unknowns $\boldsymbol{x}$ from the data $\boldsymbol{y}$ in a special case of linear inverse problems $\boldsymbol{y}=\boldsymbol{A x}+\boldsymbol{n}$ using a full bayesian approach and presented four algorithms to estimate simultanously the hyperparameters $\theta$ and $\beta$ and the unknowns $\boldsymbol{x}$. The main structure of all of these algorithms are the same even if the procedure to deduce them have been different. However, we have not yet really tested them to give any conclusion about their relative performances. Note however that one of them distinguishes itself from the others by taking account of the measurement system operator $\boldsymbol{A}$ and the covariance structure $\boldsymbol{P}_{0}$ of $\boldsymbol{x}$ in the hyperparameters updating step and, by the same way, by its calculation cost. We hope to be able to give some measure of their relative performances in simulation and in real applications in near future.

\section{References}

1. G. Box and G.C. Tiao, Bayesian inference in statistical analysis. Addison-Wesley publishing, 1972 .

2. H. Sorenson, Parameter estimation. Marcel Dekker, Inc., 1980.

3. J. Besag, "Digital image processing: Towards Bayesian image analysis," Journal of Applied Statistics, vol. 16, no. 3, pp. 395-407, 1989.

4. P. J. Green, "Bayesian reconstructions from emission tomography data using a modified EM algorithm," IEEE Transactions on Medical Imaging, vol. 9, pp. 84-93, Mar. 1990.

5. D. Malec and J. Sedransk, "Bayesian methodology for combining the results from different experiments when the specifications for pooling are uncertain," Biometrika, vol. 79, no. 3, pp. 593-601, 1992.

6. G. Gindi, M. Lee, A. Rangarajan, and Z. I., "Bayesian reconstruction of functional images using anatomical information as priors," IEEE Transactions on Medical Imaging, vol. MI12, no. 4, pp. 670-680, 1993.

7. J. Bernardo and A. Smith, Bayesian Theory. Chichester, England: John Wiley, 1994.

8. Barndorff-Nielsen, Information and Exponential Model in Statistics. New-York: John Wiley, 1978.

9. H. Derin, H. Elliott, R. Cristi, and D. Geman, "Bayes smoothing algorithms for segmentation of binary images modeled by markov random fields," IEEE Transactions on Pattern Analysis and Machine Intelligence, vol. PAMI-6, p. 4, 1984.

10. S. Geman and D. Geman, "Stochastic relaxation, Gibbs distributions, and the Bayesian restoration of images," IEEE Transactions on Pattern Analysis and Machine Intelligence, vol. PAMI-6, p. 2, 1984. 
11. A. Tarantola, Inverse problem theory: Methods for data fitting and model parameter estimation. Amsterdam: Elsevier Science Publishers, 1987.

12. J. Skilling, Maximum-Entropy and Bayesian Methods. Dordrecht, The Netherlands: Kluwer Academic Publisher, 1988.

13. Titterington and Rossi, "Another look at a Bayesian direct deconvolution method," Signal Processing, vol. 9, pp. 101-106, 1985.

14. G. Demoment, "Image reconstruction and restoration : Overview of common estimation structure and problems," IEEE Transactions on Acoustics Speech and Signal Processing, vol. 37, pp. 2024-2036, Dec. 1989.

15. K.-Y. Liang and D. Tsou, "Empirical Bayes and conditional inference with many nuisance parameters," Biometrika, vol. 79, no. 2, pp. 261-270, 1992.

16. R. E. McCulloch and P. E. Rossi, "Bayes factors for nonlinear hypotheses and likelihood distributions," Biometrika, vol. 79, no. 4, pp. 663-676, 1992.

17. J. Idier and Y. Goussard, "Markov modeling for Bayesian restoration of two-dimensional layered structures," IEEE Transactions on Information Theory, vol. 39, pp. 1356-1373, July 1993.

18. A. Mohammad-Djafari, "On the estimation of hyperparameters in Bayesian approach of solving inverse problems," in Proceedings of IEEE ICASSP, pp. 567-571, 1993.

19. A. Nallanathan and W. J. Fitzgerald, "Bayesian model selection applied to spatial signal processing," Proceedings of the IEE, vol. 141, pp. 76-80, Feb. 1994.

20. J. Diebolt and C. P. Robert, "Estimation of finite mixture distributions through Bayesian sampling," Journal of Royal Statistical Society B, vol. 56, no. 2, pp. 363-375, 1994.

21. H. Carfantan and A. Mohammad-Djafari, "A Bayesian approach for nonlinear inverse scattering tomographic imaging," in Proceedings of IEEE ICASSP, vol. IV, pp. 2311-2314, May 1995.

22. J. Cullum, "The effective choice of the smoothing norm in regularization," Math. Comp., vol. 33 , pp. 149-170, 1979.

23. Titterington, "General structure of regularization procedures in image reconstruction," $A s$ trononmy and Astrophysics, vol. 144, pp. 381-387, 1985.

24. L. Younès, "Estimation and annealing for Gibbsian fields," Annales de l'institut Henri Poincaré, vol. 24, pp. 269-294, Feb. 1988.

25. S. Lakshmanan and H. Derin, "Simultaneous parameter estimation and segmentation of Gibbs random fields using simulated annealing," IEEE Transactions on Pattern Analysis and Machine Intelligence, vol. PAMI-11, no. 8, pp. 799-813, 1989.

26. A. Mohammad-Djafari and J. Idier, "Maximum likelihood estimation of the lagrange parameters of the maximum entropy distributions," in Maximum Entropy and Bayesian Methods in Science and Engineering (C. Smith, G. Erikson, and P. Neudorfer, eds.), pp. 131-140, Kluwer Academic Publishers, 1991.

27. Thompson, Brown, Kay, and Titterington, "A study of methods of choosing the smoothing parameter in image restoration by regularization," IEEE Transactions on Pattern Analysis and Machine Intelligence, vol. 13, Apr. 1991.

28. E. Gassiat, F. Monfront, and Y. Goussard, "On simultaneous signal estimation and parameter identification using a generalized likelihood approach," IEEE Transactions on Information Theory, vol. IT-38, pp. 157-162, 1992.

29. T. J. Hebert and R. Leahy, "Statistic-based map image reconstruction from poisson data using Gibbs prior," IEEE trans. on Signal Processing, vol. 40, pp. 2290-2303, Sept. 1992.

30. C. Bouman and K. Sauer, "Maximum likelihood scale estimation for a class of markov random fields penalty for image regularization," in Proceedings of IEEE ICASSP, vol. V, pp. 537-540, 1994.

31. A. N. Iusem and B. F. Svaiter, "A new smoothing-regularization approach for a maximumlikelihood problem," Applied Mathematics and Optimization, vol. 29, pp. 225-241, 1994.

32. A. Dempster, N. Laird, and D. Rubin, "Maximum likelihood from incomplete data via the EM algorithm," Journal of Royal Statistical Society B, vol. 39, pp. 1-38, 1977.

33. Vardi and Lee, "From image deblurring to optimal investments maximum likelihood solutions for positive linear inverse problems," Journal of Royal Statistical Society B, vol. 55, no. 3, pp. 569-612, 1993. 